\title{
BIHARMONIC MAPS ON V-MANIFOLDS
}

\author{
YUAN-JEN CHIANG and HONGAN SUN
}

(Received 16 February 2001)

\begin{abstract}
We generalize biharmonic maps between Riemannian manifolds into the case of the domain being V-manifolds. We obtain the first and second variations of biharmonic maps on V-manifolds. Since a biharmonic map from a compact V-manifold into a Riemannian manifold of nonpositive curvature is harmonic, we construct a biharmonic non-harmonic map into a sphere. We also show that under certain condition the biharmonic property of $f$ implies the harmonic property of $f$. We finally discuss the composition of biharmonic maps on V-manifolds.
\end{abstract}

2000 Mathematics Subject Classification. 58E20, 35K05.

1. Introduction. Following Eells, Sampson, and Lemaire's tentative ideas [7, 8, 9], Jiang first discussed biharmonic (or 2-harmonic) maps between Riemannian manifolds in his two articles $[10,11]$ in China in 1986, which gives the conditions for biharmonic maps. A biharmonic map $f: M \rightarrow N$ between Riemannian manifolds is the smooth critical point of the bi-energy functional

$$
E_{2}(f)=\int_{M}\left\|\left(d+d^{*}\right) f\right\|^{2} * 1=\int_{M}\|\boldsymbol{\tau}(f)\|^{2} * 1,
$$

where $* 1$ is the volume form on $M$, the tension field $\tau(f)=(\hat{D} d f)\left(e_{i}, e_{i}\right)\left(=\left(\hat{D}_{e_{i}} d f\right)\left(e_{i}\right)\right)$, $\left\{e_{i}\right\}$ is the local frame of a point $p$ in $M$. Biharmonic maps are the extensions of harmonic maps, and their study provides a source in partial differential equations, differential geometry, and analysis. After Jiang, Chiang, and Sun have studied biharmonic maps in two papers $[6,14]$. Chiang also studied harmonic maps and biharmonic maps of two different kinds of singular spaces: V-manifolds [3, 4] and spaces with conical singularities (with Andrea Ratto [5]).

In this paper, we generalize the notion of a biharmonic map to the case that the domain of $f$ is a V-manifold due to Satake in $[1,12,13]$. A $\left(C^{\infty}\right) \mathrm{V}$-manifold $(M, \mathscr{F})$ consists of a Hausdorff space $M$ with an atlas $\mathscr{F}$ of V-charts satisfying the following conditions:

(i) If $\{\tilde{U}, G, \pi\}$ and $\left\{\tilde{U}^{\prime}, G^{\prime}, \pi^{\prime}\right\}$ are two V-charts in $\mathscr{F}$ over $U, U^{\prime}$, respectively, in $M$ with $U \subset U^{\prime}$, then there exists an injection $\lambda:\{U, G, \pi\} \rightarrow\left\{U, G^{\prime}, \pi^{\prime}\right\}$.

(ii) The supports of V-charts in $\mathscr{F}$ form a basis for open sets in $M$.

Take a chart $\{\tilde{U}, G, \pi\} \in \mathscr{F}_{\mathrm{F}}$ such that $p \in \pi(\tilde{U})$ and choose $\tilde{p} \in \tilde{U}$ such that $\sigma \tilde{p}=\tilde{p}$. The isotropic subgroup $G_{\tilde{p}}$ of $G$ at $\tilde{p}$ is the set of all $\sigma \in G$ such that $\sigma \tilde{p}=\tilde{p}$. So $G_{\tilde{p}}$ is called the isotropic group of $p$. The singular set $\mathbb{S}$ of $M$ consists of all singular points of $M$, that is, the points of $M$ with nontrivial isotropy groups. (For example, $S^{2} / Z_{3}$ is a compact V-manifold with two singular points.) The main difficulties of this 
paper arise from the complicated behavior of the singular locus of V-manifolds, and therefore a different method than the usual one is required. In fact, this article is the extension of Chiang's previous two papers [3, 4].

We derive the first variations of biharmonic maps in Theorem 2.2, and give the definition for biharmonic maps on $\mathrm{V}$-manifolds. We show that a biharmonic map from a compact V-manifold into a Riemannian manifold of nonpositive curvature is a harmonic map in Theorem 2.4. Then we construct a biharmonic non-harmonic map from a V-manifold into a sphere in Section 2. We obtain the second variations of biharmonic maps in Theorem 3.1. If $d^{2} /\left.d t^{2} E_{2}\left(f_{t}\right)\right|_{t=0} \geq 0$, then $f$ is a stable biharmonic map. In Theorem 3.3, we show that if a stable biharmonic map from a compact V-manifold $M$ into a Riemannian manifold $N$ of positive curvature satisfies the conservation law, then $f$ must be a harmonic map. In Theorem 3.4, we prove the composition of biharmonic maps on V-manifolds which generalizes Sun's result in [14].

2. Biharmonic maps on V-manifolds. Let $(M, \mathscr{F})$ be a $\left(\mathbb{C}^{\infty}\right) \mathrm{V}$-manifold, and $U$ be an open subset of $M$. By a $V$-chart on $M$ over $U$ we mean a system $\{\tilde{U}, G, \pi\}$ consisting of (1) a connected open subset $\tilde{U}$ of $\mathbb{R}^{m}$, (2) a finite group $G$ of diffeomorphisms of $\tilde{U}$, with the set of fixed points of codimension $\geq 2$, and (3) a continuous map of $\pi: \tilde{U} \rightarrow U$ such that $\pi \circ \sigma=\pi$ for $\sigma \in G$ and such that $\pi$ induces a homeomorphism of $\tilde{U} / G$ onto $U$. The set $U$ is called the support of V-chart, and $\pi$ is called the projection onto $U$.

Let $(M, \mathscr{F})$ be a V-manifold and $p \in M$. Take a chart $\{\tilde{U}, G, \pi\} \in \mathscr{F}$ such that $p \in$ $\pi(\tilde{U})$ and choose $\tilde{p} \in \tilde{U}$ such that $\pi(\tilde{p})=p$. The isotropic subgroup $G_{\tilde{p}}$ of $G$ at $\tilde{p}$ is the set of all $\sigma \in G$ such that $\sigma \tilde{p}=\tilde{p}$, and is uniquely determined by $p$. Therefore, $G_{\tilde{p}}$ is called the isotropic group of $p$. The singular set $\mathbb{S}$ of $M$ consists of all singular points of $M$, that is, the points of $M$ with nontrivial isotropic groups. Let $\left(\tilde{x}^{1}, \ldots, \tilde{x}^{m}\right)$ be a coordinate system around $\tilde{p}$ and consider the system $\tilde{y}^{i}=1 /\left|G_{\tilde{p}}\right| \sum l_{i j}\left(\sigma^{-1}\right) \tilde{x}^{j} \cdot \sigma$ with

$$
l_{i j}(\sigma)=\left[\frac{\partial \tilde{x}^{i} \circ \sigma}{\partial \tilde{x}^{j}}\right]_{\tilde{p}}, \quad\left|G_{\tilde{p}}\right|=\text { order of } G_{\tilde{p}} .
$$

Then the $\left\{\tilde{y}^{i}\right\}$ are a new coordinate system around $\tilde{p}$ and $G_{\tilde{p}}$ operates linearly in the $\tilde{y}$-system. After this suitable $C^{\infty}$ change of coordinates around $\tilde{p}, G_{\tilde{p}}$ becomes a finite group of linear transformations. The fixed point set of any $\sigma \in G_{\tilde{p}}$ is the defined linear equations in the $\tilde{y}$, and consequently the fixed point set of $\sigma \in G_{\tilde{p}}$ in $\tilde{U}$ is the intersection of $\tilde{U}$ with a linear space. Therefore, $\pi^{-1} \mathbb{S}$ is locally expressed by a finite union of linear spaces intersected with $\tilde{U}$. Hence $\mathbb{S}$ is a V-submanifold of codimension $\geq 2$ of $M$. Clearly, $M-\mathbb{S}$ is an ordinary manifold.

We fix a V-manifold $M$ with defining atlas $\mathscr{F}$. A smooth function $f:(M, \mathscr{F}) \rightarrow N$ from $M$ into an ordinary manifold $N$ is defined as follows: for any $\{\tilde{U}, G, \pi\} \in \mathscr{F}_{\text {f }}$ there corresponds an ordinary $G$-invariant smooth map $f_{\tilde{U}}^{G}=1 /|G| \sum_{\sigma \in G} f_{\tilde{U}} \circ \sigma: \tilde{U} \rightarrow N$ such that $f_{\tilde{U}}^{G}=f \circ \pi$ and $f_{\tilde{U}}^{G}=f_{\tilde{U}^{\prime}}^{G^{\prime}} \circ \lambda$ for any injection $\lambda:\{\tilde{U}, G, \pi\} \rightarrow\left\{\tilde{U}^{\prime}, G^{\prime}, \pi^{\prime}\right\}$ where $f_{\tilde{U}}: \tilde{U} \rightarrow N$ is an ordinary smooth map.

Put a Riemannian metric $g_{\tilde{U}}=g_{i j} d \tilde{x}^{i} d \tilde{x}^{j}$ on $\tilde{U}$. By taking the $G$-average if necessary, we can assume that $g_{\tilde{U}}$ is $G$-invariant. Thus the transformations $\sigma \in G$ are isometries for $g_{\tilde{U}}$. By using the standard partition of unity construction, we can patch all such 
local invariant metrics together into a global metric tensor field of type $(0,2)$ on the V-manifold $M$, which we call a Riemannian metric on $M$.

Let $M^{m}$ be a compact V-manifold of dimension $m$ with $\mathbb{C}^{\infty}$ Riemannian metric $\mathfrak{g}$, and $N^{n}$ a $\left(\mathbb{C}^{\infty}\right)$ Riemannian manifold of dimension $n$. By Satake [12, 13], $M$ admits a finite triangulation $T=\cup s_{\alpha}$ such that each $s_{\alpha}$ is contained in the support $U_{\alpha}$ of a Vchart $\left\{\tilde{U}_{\alpha}, G_{\alpha}, \pi_{\alpha}\right\} \in \mathscr{F}_{F}$ on $M$ and is the homeomorphic projection of a regular simplex $\tilde{s}_{\alpha}$ in $\tilde{U}_{\alpha}$. For a smooth map $f: M \rightarrow N$, the bi-energy functional of $f$ is defined by

$$
E_{2}(f)=\int_{M}|\tau(f)|^{2} * 1=\sum \int_{s_{\alpha}}|\tau(f)|^{2} d x_{\alpha}=\sum \frac{1}{\left|G_{\alpha}\right|} \int_{\tilde{s}_{\alpha}}|\tau(\tilde{f})|^{2} d \tilde{x}_{\alpha}
$$

where $d \tilde{x}_{\alpha}$ denotes the volume form with respect to the $G_{\alpha}$-invariant metric $g_{i j}$ in $\tilde{U}_{\alpha}$, $\tilde{f}_{\alpha}: \tilde{U}_{\alpha} \rightarrow N$ is the $G_{\alpha}$-invariant lift of $f$. The Green's divergence theorem on a compact $\mathrm{V}$-manifold proved in [3] plays an important role in the proofs of both Theorems 2.2 and 3.1.

In order to compute the Euler-Lagrange equation, we consider a one-parameter family of maps $\left\{f_{t}\right\} \in \mathbb{C}^{\infty}(M, N), t \in I_{\epsilon}=(-\epsilon, \epsilon), \epsilon>0$ such that in the V-chart $\{\tilde{U}, G, \pi\} \in \mathscr{F}$ over the support $U$ on $M$, the $G$-invariant lift $\tilde{f}_{t}$ is the endpoint of the segment starting at $G$-invariant lift $\widetilde{f(x)}$ determined in length and direction by the vector field $\dot{\tilde{f}}$ along $\tilde{f}$, and such that $\partial \tilde{f}_{t} / \partial t=0$ and $\bar{D}_{\tilde{e}_{i}} \partial \tilde{f}_{t} / \partial t=0$ outside a compact subset of the interior of $\tilde{U}$. Choose $\left\{e_{i}\right\}$ being the local frame of a point $p$ in $U$ on $M$, and $\left\{\tilde{e}_{i}\right\}$ being the local frame of the lifting point $\tilde{p}$ in $\tilde{U}$. Let $D, D^{\prime}, \bar{D}, \hat{D}$ be the Riemannian connections along $T M, T N, f^{-1} T N, T^{*} M \otimes f^{-1} T N$, and $\tilde{D}, \tilde{\hat{D}}$ are the Riemannian connections along $T \tilde{U}, T^{*} \tilde{U} \otimes f^{-1} T N$ in each $\{\tilde{U}, G, \pi\} \in \mathscr{F}$ over the support $U$ on $M$. Also, let $\triangle=\bar{D}_{\tilde{e}_{k}} \bar{D}_{\tilde{e}_{k}}-\bar{D}_{D_{\tilde{e}_{k}} \tilde{e}_{k}}$ be the Laplace operator along the cross section of $f^{-1} T N$ in each $\tilde{U}$, and $V=\partial \tilde{f}_{t} / \partial t$. We can compute (2.2) directly, and obtain the following result.

LEMMA 2.1.

$$
\begin{aligned}
\frac{d}{d t} E_{2}\left(f_{t}\right)= & 2 \Sigma \frac{1}{\left|G_{\alpha}\right|} \int_{\tilde{s}_{\alpha}}\left\langle\tilde{\hat{D}}_{\tilde{e}_{i}} \tilde{\hat{D}}_{\tilde{e}_{i}} d \tilde{f}_{t}\left(\frac{\partial}{\partial t}\right)-\tilde{\hat{D}}_{\tilde{D}_{\tilde{e}_{i}} \tilde{e}_{i}} d \tilde{f}_{t}\left(\frac{\partial}{\partial t}\right),\left(\tilde{\hat{D}}_{\tilde{e}_{j}} d \tilde{f}_{t}\right)\left(\tilde{e}_{j}\right)\right\rangle d \tilde{x}_{\alpha} \\
& +2 \Sigma \frac{1}{\left|G_{\alpha}\right|} \int_{\tilde{s}_{\alpha}}\left\langle R^{N}\left(d \tilde{f}_{t}\left(\tilde{e}_{i}\right), d \tilde{f}_{t}\left(\frac{\partial}{\partial t}\right)\right) d \tilde{f}_{t}\left(\tilde{e}_{i}\right),\left(\tilde{\hat{D}}_{\tilde{e}_{j}} d \tilde{f}_{t}\right)\left(\tilde{e}_{j}\right)\right\rangle d \tilde{x}_{\alpha} .
\end{aligned}
$$

THEOREM 2.2. Let $f:(M, \mathscr{F}) \rightarrow N$ be a smooth map from a compact V-manifold $(M, \mathscr{F})$ into a Riemannian manifold $N$. Set $V=\partial \tilde{f}_{t} / \partial t$ then

$$
\left.\frac{d}{d t}\right|_{t=0} E_{2}\left(f_{t}\right)=2 \sum \frac{1}{\left|G_{\alpha}\right|} \int_{\tilde{s}_{\alpha}}\left\langle V, \triangle \boldsymbol{T}(\tilde{f})+R^{N}\left(d \tilde{f}\left(\tilde{e}_{i}\right), \tau(\tilde{f})\right) d \tilde{f}\left(\tilde{e}_{i}\right)\right\rangle d \tilde{x}_{\alpha} .
$$

Proof. For every $t \in I_{\epsilon}$, let

$$
\tilde{X}=\left\langle\tilde{\hat{D}}_{\tilde{e}_{i}} d \tilde{f}_{t}\left(\frac{\partial}{\partial t}\right), \tilde{\hat{D}}_{\tilde{e}_{j}} d \tilde{f}_{t}\left(\tilde{e}_{j}\right)\right\rangle \tilde{e}_{i}, \quad \tilde{Y}=\left\langle d \tilde{f}_{t}\left(\frac{\partial}{\partial t}\right), \bar{D}_{\tilde{e}_{i}}\left(\tilde{\hat{D}}_{\tilde{e}_{j}} d \tilde{f}_{t}\right)\left(\tilde{e}_{j}\right)\right\rangle\left(\tilde{e}_{i}\right),
$$

in each $\{\tilde{U}, \pi, G\} \in \mathscr{F}$ over the support $U$ on $M$. By computing the divergence of $\tilde{X}$ and $\tilde{Y}$ in each $\tilde{U}$, and applying Green's divergence theorem to the vector field $\tilde{X}-\tilde{Y}$ 
in each $\tilde{\triangle}$ on the compact manifold $M$ in [3], we have

$$
\begin{aligned}
& \sum \frac{1}{\left|G_{\alpha}\right|} \int_{\tilde{s}_{\alpha}}\left\langle\left(\tilde{\hat{D}}_{\tilde{e}_{i}} \tilde{\hat{D}}_{\tilde{e}_{i}} d \tilde{f}_{t}\right)\left(\frac{\partial}{\partial t}\right)-\left(\tilde{\hat{D}}_{\tilde{D}_{\tilde{e}_{i}} \tilde{e}_{i}} d \tilde{f}_{t}\right)\left(\frac{\partial}{\partial t}\right),\left(\tilde{\tilde{D}}_{\tilde{e}_{j}} d \tilde{f}_{t}\right)\left(\tilde{e}_{j}\right)\right\rangle d \tilde{x}_{\alpha} \\
& \quad=\sum \frac{1}{\left|G_{\alpha}\right|} \int_{\tilde{s}_{\alpha}}\left\langle d \tilde{f}_{t}\left(\frac{\partial}{\partial t}\right), \bar{D}_{\tilde{e}_{k}} \bar{D}_{\tilde{e}_{k}}\left(\tilde{\hat{D}}_{\tilde{e}_{j}} d \tilde{f}_{t}\right)\left(\tilde{e}_{j}\right)-\bar{D}_{\tilde{D}_{\tilde{e}_{k}} \tilde{e}_{k}}\left(\left(\tilde{\hat{D}}_{\tilde{e}_{j}} d \tilde{f}_{t}\right)\left(\tilde{e}_{j}\right)\right)\right\rangle d \tilde{x}_{\alpha} .
\end{aligned}
$$

By the assumption, $\partial \tilde{f}_{t} / \partial t=0$ and $\bar{D}_{\tilde{e}_{i}} \partial \tilde{f}_{t} / \partial t=0$ outside of the compact subset of the interior of each $\tilde{U}$, and substituting (2.6) into (2.3), we get

$$
\begin{aligned}
\left.\frac{d}{d t}\right|_{t=0} E_{2}\left(f_{t}\right)= & 2 \sum \frac{1}{\left|G_{\alpha}\right|} \int_{\tilde{s}_{\alpha}}\left\langle d \tilde{f}_{t}\left(\frac{\partial}{\partial t}\right), \bar{D}_{\tilde{e}_{k}} \bar{D}_{\tilde{e}_{k}}\left(\tilde{\hat{D}}_{\tilde{e}_{j}} d \tilde{f}_{t}\right)\left(\tilde{e}_{j}\right)\right. \\
& \left.-\bar{D}_{\tilde{D}_{\tilde{e}_{k}} \tilde{e}_{k}}\left(\left(\tilde{\hat{D}}_{\tilde{e}_{j}} d \tilde{f}_{t}\right)\left(\tilde{e}_{j}\right)\right)\right\rangle d \tilde{x}_{\alpha} \\
& +2 \sum \frac{1}{\left|G_{\alpha}\right|} \int_{\tilde{s}_{\alpha}}\left\langle R^{N}\left(d \tilde{f}_{t}\left(\tilde{e}_{i}\right), d \tilde{f}_{t}\left(\frac{\partial}{\partial t}\right)\right) d \tilde{f}_{t}\left(\tilde{e}_{i}\right),\left(\tilde{\hat{D}}_{\tilde{e}_{j}} d \tilde{f}_{t}\right)\left(\tilde{e}_{j}\right)\right\rangle d \tilde{x}_{\alpha} .
\end{aligned}
$$

Let $t=0$, and by the symmetry of the Riemannian curvature tensor, we derive (2.4).

DEFINITION 2.3. A smooth map $f:(M, \mathscr{F}) \rightarrow N$ from a compact V-manifold $M$ into a Riemannian manifold $N$ is biharmonic if and only if

$$
\boldsymbol{\tau}_{2}(\tilde{f})=\triangle \boldsymbol{\tau}(\tilde{f})+R^{N}\left(d \tilde{f}\left(\tilde{e}_{i}\right), \tau(\tilde{f})\right) d \tilde{f}\left(\tilde{e}_{i}\right)=0
$$

in each $\{\tilde{U}, G, \pi\} \in \mathscr{F}$ over the support $U$ on $M$.

A harmonic map $f: M \rightarrow N$ on a $\mathrm{V}$-manifold $M$ is obviously a biharmonic map, but a harmonic map is not necessarily a biharmonic map. However, we obtain the following theorem.

THEOREM 2.4. Suppose that $M$ is a compact $V$-manifold, and $N$ is a Riemannian manifold of nonpositive curvature. If $f: M \rightarrow N$ is a biharmonic map, then $f$ is a harmonic map.

Proof. In each V-chart $\{\tilde{U}, G, \pi\} \in \mathscr{F}$ over the support $U$ on $M$ it is calculated by

$$
\begin{aligned}
\triangle e_{2}(\tilde{f}) & =\frac{1}{2} \triangle\|\tau(\tilde{f})\|^{2}=\left\langle\tilde{D}_{\tilde{e}_{k}} \tau(\tilde{f}), \tilde{D}_{\tilde{e}_{k}} \tau(\tilde{f})\right\rangle+\left\langle\bar{D}^{*} \bar{D} \tau(\tilde{f}), \tau(\tilde{f})\right\rangle \\
& =\left\langle\tilde{D}_{\tilde{e}_{k}} \tau(\tilde{f}), \tilde{D}_{e_{k}} \tau(\tilde{f})\right\rangle-\left\langle R^{N}\left(d \tilde{f}\left(\tilde{e}_{i}\right), \tau(\tilde{f})\right) d \tilde{f}\left(\tilde{e}_{i}\right), \tau(\tilde{f})\right\rangle \geq 0,
\end{aligned}
$$

because $\tau_{2}(\tilde{f})=0$ in each $\tilde{U}$ and the Riemannian curvature of $N$ is nonpositive. By Bochner's technique and the assumption $\partial \tilde{f}_{t} / \partial t=0$ and $\bar{D}_{\tilde{e}_{i}} \partial \tilde{f}_{t} / \partial t=0$ outside a compact subset of $\operatorname{int}(\tilde{U})$, we know $\|\tau(\tilde{f})\|^{2}=$ const, and then substituting into (2.9) we have $\bar{D}_{\tilde{e}_{k}}(\tau \tilde{f})=0$, for all $k=1,2, \ldots, m$ by [7] which implies $\tau(\tilde{f})=0$ in each $\tilde{U}$, that is, $f$ is harmonic on $M$.

Since harmonic maps are automatically biharmonic maps when the Riemannian curvature of $N$ is nonpositive, we will find a non-trivial biharmonic map into a sphere. By the concepts of V-manifolds and the similar techniques as [11], we have the following theorem. 
THEOREM 2.5. Let $f:(M, \mathscr{F}) \rightarrow S^{m+1}$ be nonzero parallel mean curvature isometric embedding, then $f$ is biharmonic if and only if the second fundamental form $B(\tilde{f})$ of $\tilde{f}$ with $\|B(\tilde{f})\|^{2}=m=\operatorname{dim}(\tilde{U})$ in each $\tilde{U}$ over the support $U$ on $M$.

EXAMPLE 2.6. In $S^{m+1}$, the compact hypersurface of its Gauss map being isometric embedding is the Clifford surface (see [15]):

$$
M_{k}^{m}(1)=S^{k}\left(\sqrt{\frac{1}{2}}\right) \times S^{m-k}\left(\sqrt{\frac{1}{2}}\right), \quad 0 \leq k \leq m .
$$

Let $f: M_{k}^{m}(1) \rightarrow S^{m+1}$ be the standard embedding. Set

$$
M_{k}^{m}(1)^{\prime}=\frac{S^{k}(\sqrt{1 / 2})}{Z_{p}} \times \frac{S^{m-k}(\sqrt{1 / 2})}{Z_{p^{\prime}}}
$$

where $p, p^{\prime}$ are prime numbers ( $p$ and $p^{\prime}$ could be the same or different). Since both the first and the second terms are compact V-manifolds, the product is also a compact V-manifold. Let $f^{\prime}: M_{k}^{m}(1)^{\prime} \rightarrow S^{m+1}$ be a map such that $k \neq m / 2$, pick $\tilde{U}=\left\{\left(x^{0}, x^{1}, \ldots, x^{k}\right) \in S^{k} \sqrt{1 / 2}: x^{i}>0, i\right.$ is any of $\left.0,1, \ldots, k\right\} \times\left\{\left(x^{k+1}, \ldots, x^{m+1}\right) \in\right.$ $S^{m-k} \sqrt{1 / 2}: x^{j}>0, j$ is any of $\left.k+1, \ldots, m+1\right\}$ (if $x^{i}$ and $x^{j}$ vary, $\tilde{U}$ is different), and let $\tilde{f}^{\prime}: \tilde{U} \rightarrow S^{m+1}$ (as part of the standard map $f: S^{k} \sqrt{1 / 2} \times S^{m-k} \sqrt{1 / 2} \rightarrow S^{m+1}$ ) in each $\{\tilde{U}, G, \pi\} \in \mathscr{F}$. So $\tilde{f}^{\prime}$ has parallel second fundamental form, and has parallel mean curvature and $B\left(\tilde{f}^{\prime}\right)=k+m-k=m,\left\|\tau\left(\tilde{f}^{\prime}\right)\right\|=|k-(m-k)|=2 k-m \neq 0$. That is, $\tilde{f}^{\prime}$ is biharmonic in $\tilde{U}$ for each $\{\tilde{U}, G, \pi\} \in \mathscr{F}$. Then by Theorem $2.5 f$ is a nontrivial biharmonic map on $(M, \mathscr{F})$.

3. The stability and composition of biharmonic maps on V-manifolds. Let $M$ be a compact V-manifold, and $N$ a Riemannian manifold. We continue to use the notations as in the previous sections. By applying the Green's divergence theorem on the compact V-manifold $M$ [3], the concepts of V-manifolds, and the similar techniques in [11], we can have the second variations of biharmonic maps as follows.

THEOREM 3.1. If $f:(M \mathscr{F}) \rightarrow N$ is a biharmonic map, then

$$
\begin{aligned}
\left.\frac{1}{2} \frac{d^{2}}{d t^{2}} E_{2}\left(f_{t}\right)\right|_{t=0} & \\
=\sum \frac{1}{\left|G_{\alpha}\right|} \int_{\tilde{s}_{\alpha}} \| & \Delta V+R^{N}\left(d \tilde{f}\left(\tilde{e}_{i}\right), V\right) d \tilde{f}\left(\tilde{e}_{i}\right) \|^{2} d \tilde{x}_{\alpha} \\
+\sum \frac{1}{\left|G_{\alpha}\right|} \int_{\tilde{s}_{\alpha}} & \left\langle V,\left(D^{\prime}{ }_{d \tilde{f}\left(\tilde{e}_{k}\right)} R^{N}\right)\left(d \tilde{f}\left(\tilde{e}_{k}\right), \tau(\tilde{f})\right) V\right. \\
& +\left(D^{\prime}{ }{ }_{T(\tilde{f})} R^{N}\right)\left(d \tilde{f}\left(\tilde{e}_{i}\right), V\right) d \tilde{f}\left(\tilde{e}_{i}\right)+R^{N}(\tau(\tilde{f}), V) \tau(\tilde{f}) \\
& \left.+2 R^{N}\left(d \tilde{f}\left(\tilde{e}_{k}\right), V\right) \bar{D}_{\tilde{e}_{k}} \tau(\tilde{f})+2 R^{N}\left(d \tilde{f}\left(\tilde{e}_{i}\right), \tau(\tilde{f})\right) \bar{D}_{\tilde{e}_{i}} V\right\rangle d \tilde{x}_{\alpha} .
\end{aligned}
$$

DEFINITION 3.2. Let $f:(M, \mathscr{F}) \rightarrow N$ be a biharmonic map from a compact V-manifold $M$ into a Riemannian manifold $N$. If $d^{2} /\left.d t^{2} E_{2}\left(f_{t}\right)\right|_{t=0} \geq 0$, then $f$ is a stable biharmonic map. 
If we look at a harmonic map as a biharmonic map, then it must be stable by the definition of bi-energy since

$$
\left.\frac{1}{2} \frac{d^{2}}{d t^{2}} E_{2}\left(f_{t}\right)\right|_{t=0}=\sum \frac{1}{\left|G_{\alpha}\right|} \int_{\tilde{s}_{\alpha}}\left\|\Delta V+R^{N}(d \tilde{f})\left(\left(\tilde{e}_{i}\right), V\right) d \tilde{f}\left(\tilde{e}_{i}\right)\right\|^{2} d \tilde{x}_{\alpha} \geq 0 .
$$

THEOREM 3.3. Let $f:(M, \mathscr{F}) \rightarrow N$ be a stable biharmonic map from a compact $V$ manifold $M$ into a Riemannian manifold $N$ of constant sectional curvature $K>0$ and $f$ satisfies the conservation law, then $f$ must be a harmonic map.

Proof. Because $N$ has the constant sectional curvature, the term of $D^{\prime} R^{N}$ of the second variation formula disappears and

$$
\begin{aligned}
\left.\frac{1}{2} \frac{d^{2}}{d t^{2}} E_{2}\left(f_{t}\right)\right|_{t=0}= & \sum \frac{1}{\left|G_{\alpha}\right|} \int_{\tilde{s}_{\alpha}}\left\|\Delta V+R^{N}\left(d f\left(e_{i}\right), V\right) d f\left(e_{i}\right)\right\|^{2} d \tilde{x}_{\alpha} \\
& +\sum \frac{1}{\left|G_{\alpha}\right|} \int_{\tilde{s}_{\alpha}}\left\langle V, R^{N}(\tau(\tilde{f}), V) \tau(\tilde{f})+2 R^{N}\left(d \tilde{f}\left(\tilde{e}_{k}\right), V\right) \bar{D}_{\tilde{e}_{k}} \tau(\tilde{f})\right. \\
& \left.+2 R^{N}\left(d \tilde{f}\left(\tilde{e}_{i}\right), \tau(\tilde{f})\right) \bar{D}_{\tilde{e}_{i}} V\right\rangle d \tilde{x}_{\alpha} .
\end{aligned}
$$

Take $V=\tau(\tilde{f})$, and notice that $f$ is biharmonic and $N$ has the constant sectional curvature, then by (3.3) we have

$$
\begin{aligned}
\left.\frac{1}{2} \frac{d^{2}}{d t^{2}} E_{2}\left(f_{t}\right)\right|_{t=0}=\sum \frac{4}{\left|G_{\alpha}\right|} \int_{\tilde{s}_{\alpha}}\left\langle R^{N}\left(d \tilde{f}\left(\tilde{e}_{i}\right), \tau(\tilde{f})\right) \bar{D}_{\tilde{e}_{k}} \tau(\tilde{f}), \tau(\tilde{f})\right\rangle d \tilde{x}_{\alpha} \\
=4 K \sum \frac{1}{\left|G_{\alpha}\right|} \int_{\tilde{s}_{\alpha}}\left[\left\langle d \tilde{f}\left(\tilde{e}_{k}\right), \tilde{\bar{D}}_{\tilde{e}_{k}} \tau(\tilde{f})\right\rangle\|\tau(\tilde{f})\|^{2}\right. \\
\left.-\left\langle d \tilde{f}\left(\tilde{e}_{k}\right), \tau(\tilde{f})\right\rangle\left\langle\tau(\tilde{f}), \bar{D}_{\tilde{e}_{k}} \tau(\tilde{f})\right\rangle\right] d \tilde{x}_{\alpha} .
\end{aligned}
$$

In each $\tilde{U}_{\alpha}, \tilde{f}$ satisfies the conservation law [2], so

$$
\begin{gathered}
\left\langle d \tilde{f}\left(\tilde{e}_{k}\right), \tau(\tilde{f})\right\rangle=0, \\
\left\langle d \tilde{f}\left(\tilde{e}_{k}\right), \bar{D}_{\tilde{e}_{k}} \tau(\tilde{f})\right\rangle=-\left\langle\bar{D}_{\tilde{e}_{k}} d \tilde{f}\left(\tilde{e}_{k}\right), \tau(\tilde{f})\right\rangle=-\|\tau(\tilde{f})\|^{2}
\end{gathered}
$$

in each $\tilde{U}$. Substitute (3.5) into (3.4), and $f$ is stable, we have

$$
\left.\frac{1}{2} \frac{d^{2}}{d t^{2}} E_{2}\left(f_{t}\right)\right|_{t=0}=-4 K \sum \frac{1}{\left|G_{\alpha}\right|} \int_{\tilde{s}_{\alpha}}\|\tau(\tilde{f})\|^{4} d \tilde{x}_{\alpha} \geq 0 .
$$

Therefore, $\tau(\tilde{f})=0$ in each $\tilde{s}_{\alpha}$ of $\tilde{U}_{\alpha}$, that is, $f$ is harmonic on $(M, \mathscr{F})$.

Let $f:(M, \mathscr{F}) \rightarrow M^{\prime}$ be a smooth map from a compact V-manifold $(M, \mathscr{F})$ into a Riemannian manifold and $M^{\prime}$, and $f_{1}: M^{\prime} \rightarrow M^{\prime \prime}$ a smooth map from $M^{\prime}$ into another Riemannian manifold $M^{\prime \prime}$. Then the composition $f_{1} \circ f: M \rightarrow M^{\prime \prime}$ is a smooth map. Let $D, D^{\prime}, \bar{D}, \bar{D}^{\prime} \hat{D}, \hat{D}^{\prime}, \hat{D}^{\prime \prime}$ be the Riemannian connections on $T M, T M^{\prime}, f^{-1} T M, f_{1}^{-1} T M^{\prime \prime}$, $\left(f_{1} \circ f\right)^{-1} T M^{\prime \prime}, T^{*} M \otimes f^{-1} T M^{\prime}, T^{*} M^{\prime} \otimes f_{1}^{-1} T M^{\prime \prime}, T^{*} M \otimes\left(f_{1} \circ f\right)^{-1} T M^{\prime \prime}$, respectively, and let $R^{M^{\prime}}\left(\right.$, ),$R^{f_{1}^{-1} T M^{\prime \prime}}$ be the Riemannian curvatures on $T M^{\prime \prime}, f^{-1} T M^{\prime \prime}$, respectively. For all $X, Y \in \Gamma(T M)$, we have

$$
\bar{D}_{X}^{\prime \prime} d\left(f_{1} \circ f\right) Y=\hat{D}_{d f(X)}^{\prime} d f_{1}(Y)+d f_{1} \circ \bar{D}_{X} d f(Y) .
$$


THEOREM 3.4. Let $(M, \mathscr{F})$ be a compact $V$-manifold, and $M^{\prime}, M^{\prime \prime}$ Riemannian manifolds. If $f: M \rightarrow M^{\prime}$ is a biharmonic map and $f_{1}: M^{\prime} \rightarrow M^{\prime \prime}$ is totally geodesic, then the composition $f_{1} \circ f: M \rightarrow M^{\prime \prime}$ is a biharmonic map.

Proof. Since $f_{1}$ is totally geodesic, that is, $\hat{D}^{\prime} d f_{1}=0$, so in each $\tilde{U}$ we have $\tau\left(f_{1} \circ \tilde{f}\right)=d f_{1} \circ \tau(\tilde{f})$ and

$$
\begin{aligned}
{\overline{D^{\prime \prime}}}^{\prime \prime *} \bar{D} \tau\left(f_{1} \circ \tilde{f}\right) & ={\overline{D^{\prime \prime}}}^{\prime \prime *} \bar{D}^{\prime \prime}\left(d f_{1} \circ \tau(\tilde{f})\right) \\
& =\bar{D}_{\tilde{e}_{k}}^{\prime \prime} \bar{D}_{\tilde{e}_{k}}^{\prime \prime}\left(d f_{1} \circ \tau(\tilde{f})\right)-\bar{D}_{\bar{D}_{\tilde{e}_{k}} \tilde{e}_{k}}^{\prime \prime}\left(d f_{1} \circ \tau(\tilde{f})\right) .
\end{aligned}
$$

By (3.7) and notice that $f_{1}$ is totally geodesic, then

$$
\begin{aligned}
\bar{D}_{\tilde{e}_{k}}^{\prime \prime}\left(d f_{1} \circ \tau(\tilde{f})\right) & =\bar{D}_{\tilde{e}_{k}}^{\prime \prime}\left(d f_{1} \circ \hat{D}_{\tilde{e}_{j}} d \tilde{f}\left(\tilde{e}_{j}\right)\right) \\
& =\left(\hat{D}_{\hat{D}_{\tilde{e}_{j}}}^{\prime} d \tilde{f}\left(\tilde{e}_{k}\right)\right. \\
& =d f_{1} \circ \bar{D}_{\tilde{e}_{k}} \tau(\tilde{f}) .
\end{aligned}
$$

So

$$
\begin{aligned}
& \bar{D}_{\tilde{e}_{k}}^{\prime \prime} \bar{D}_{\tilde{e}_{k}}^{\prime \prime}\left(d f_{1} \circ \tau(\tilde{f})\right)=\bar{D}_{\tilde{e}_{k}}^{\prime \prime}\left(d f_{1} \circ \bar{D}_{\tilde{e}_{k}} \tau(\tilde{f})\right)=d f_{1} \circ \bar{D}_{\tilde{e}_{k}} \bar{D}_{\tilde{e}_{k}} \tau(\tilde{f}), \\
& \bar{D}_{\bar{D}_{\tilde{e}_{k}} \tilde{e}_{k}}^{\prime \prime}\left(d f_{1} \circ \tau(\tilde{f})\right)=d f_{1} \circ \bar{D}_{D_{\tilde{e}_{k}} \tilde{e}_{k}} \tau(\tilde{f}) .
\end{aligned}
$$

Substituting (3.10) into (3.8), we get

$$
{\overline{D^{\prime \prime}}}^{*} \tau\left(f_{1} \circ \tilde{f}\right)=d f_{1} \circ \bar{D}^{*} \bar{D} \tau(\tilde{f}) .
$$

On the other hand,

$$
\begin{aligned}
R^{M^{\prime \prime}}\left(d\left(f_{1} \circ \tilde{f}\right)\right. & \left.\left(\tilde{e}_{i}\right), \tau\left(f_{1} \circ \tilde{f}\right)\right) d\left(f_{1} \circ f\right)\left(\tilde{e}_{i}\right) \\
= & R^{f_{1}^{-1} T M^{\prime \prime}}\left(d \tilde{f}\left(\tilde{e}_{i}\right), \tau(\tilde{f})\right) d f_{1}\left(d \tilde{f}\left(\tilde{e}_{i}\right)\right) \\
= & d f_{1} \circ R^{M^{\prime}}\left(d \tilde{f}\left(\tilde{e}_{i}\right), \tau(\tilde{f})\right) d \tilde{f}\left(\tilde{e}_{i}\right) .
\end{aligned}
$$

By (3.11) and (3.12), we have

$$
\begin{aligned}
\bar{D}^{*} \bar{D}^{\prime \prime}\left(f_{1} \circ \tilde{f}\right)+R^{M^{\prime \prime}}\left(d\left(f_{1} \circ \tilde{f}\right)\left(\tilde{e}_{i}\right), \tau\left(f_{1} \circ \tilde{f}\right)\right) d\left(f_{1} \circ \tilde{f}\right)\left(\tilde{e}_{i}\right) \\
=d f_{1} \circ\left[\bar{D}^{*} \bar{D} \tau(\tilde{f})+R^{M^{\prime}}\left(d \tilde{f}\left(\tilde{e}_{i}\right), \tau(\tilde{f})\right) d \tilde{f}\left(\tilde{e}_{i}\right)\right]
\end{aligned}
$$

in each $\tilde{U}$. Hence, if $f$ is biharmonic, then $f_{1} \circ f$ is also biharmonic.

REMARK 3.5. Theorem 3.4 generalizes the main theorem in [14] into V-manifolds. The condition of $f_{1}$ being totally geodesic cannot be weakened into harmonic or biharmonic.

ACKNOWLEDGEMENT. This project is supported by National Science Foundation of China. 


\section{REFERENCES}

[1] W. L. Baily Jr., The decomposition theorem for V-manifolds, Amer. J. Math. 78 (1956), 862-888. MR 20\#6537. Zbl 173.22705.

[2] P. Baird and J. Eells, A conservation law for harmonic maps, Geometry Symposium, Utrecht 1980 (Utrecht, 1980), Lecture Notes in Math., vol. 894, Springer, Berlin, 1981, pp. 1-25. MR 83i:58031. Zbl 485.58008.

[3] Y.-J. Chiang, Harmonic maps of V-manifolds, Ann. Global Anal. Geom. 8 (1990), no. 3, 315-344. MR 92c:58021. Zbl 679.58014.

[4] _ Spectral geometry of $V$-manifolds and its application to harmonic maps, Differential Geometry: Partial Differential Equations on Manifolds (Los Angeles, CA, 1990) (Rhode Island), Proc. Sympos. Pure Math., vol. 54, Part 1, Amer. Math. Soc., 1993, pp. 93-99. MR 94c:58040. Zbl 806.58005.

[5] Y.-J. Chiang and A. Ratto, Harmonic maps on spaces with conical singularities, Bull. Soc. Math. France 120 (1992), no. 2, 251-262. MR 93h:58040. Zbl 758.53023.

[6] Y-J. Chiang and H. Sun, 2-harmonic totally real submanifolds in a complex projective space, Bull. Inst. Math. Acad. Sinica 27 (1999), no. 2, 99-107. MR 2000e:53079. Zbl 960.53036 .

[7] J. Eells and L. Lemaire, A report on harmonic maps, Bull. London Math. Soc. 10 (1978), no. 1, 1-68. MR 82b:58033. Zbl 401.58003.

[8]__ Another report on harmonic maps, Bull. London Math. Soc. 20 (1988), no. 5, 385524. MR 89i:58027. Zbl 669.58009.

[9] J. Eells, Jr. and J. H. Sampson, Harmonic mappings of Riemannian manifolds, Amer. J. Math. 86 (1964), 109-160. MR 29\#1603. Zbl 122.40102.

[10] G. Y. Jiang, 2-harmonic isometric immersions between Riemannian manifolds, Chinese Ann. Math. Ser. A 7 (1986), no. 2, 130-144 (Chinese). MR 87k:53140. Zbl 596.53046.

[11] , 2-harmonic maps and their first and second variational formulas, Chinese Ann. Math. Ser. A 7 (1986), no. 4, 389-402 (Chinese). MR 88i:58039. Zbl 0628.58008.

[12] I. Satake, On a generalization of the notion of manifold, Proc. Nat. Acad. Sci. U.S.A. 42 (1956), 359-363. MR 18,144a. Zbl 074.18103.

[13] _ The Gauss-Bonnet theorem for V-manifolds, J. Math. Soc. Japan 9 (1957), 464-492. MR 20\#2022. Zbl 080.37403.

[14] H. Sun, A theorem on 2-harmonic mappings, J. Math. (Wuhan) 12 (1992), no. 1, 103-106 (Chinese). MR 94c:58045. Zbl 0766.53036.

[15] Y. L. Xin and X. P. Chen, The hypersurfaces in the Euclidean sphere with relative affine Gauss maps, Acta Math. Sinica 28 (1985), no. 1, 131-139 (Chinese). MR 87b:53088. Zbl 0567.53041.

Yuan-Jen Chiang: Department of Mathematics, Mary Washington College, FrederICKSBURG, VA 22401, USA

E-mail address: ychi ang@mwc. edu

Hongan Sun: Southern InStitute of Metallurgy, Ganzou, JiAngXi, China 


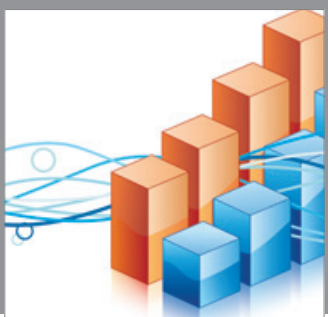

Advances in

Operations Research

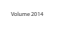

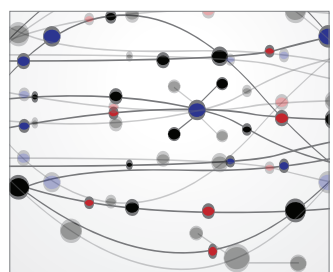

\section{The Scientific} World Journal
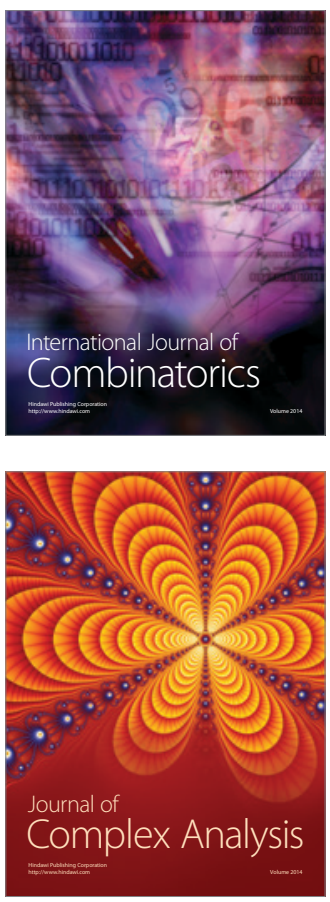

International Journal of

Mathematics and

Mathematical

Sciences
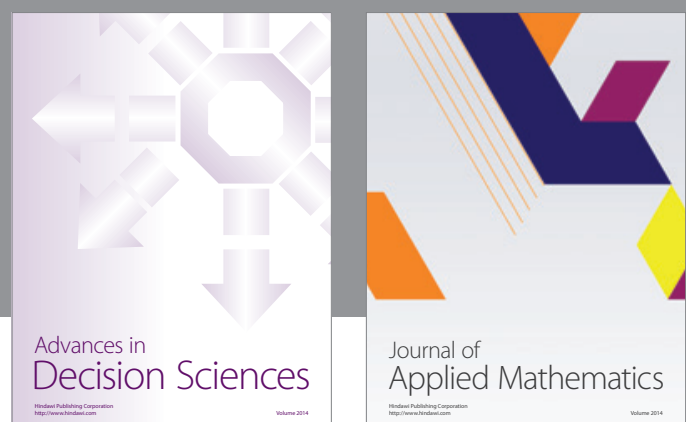

Journal of

Applied Mathematics
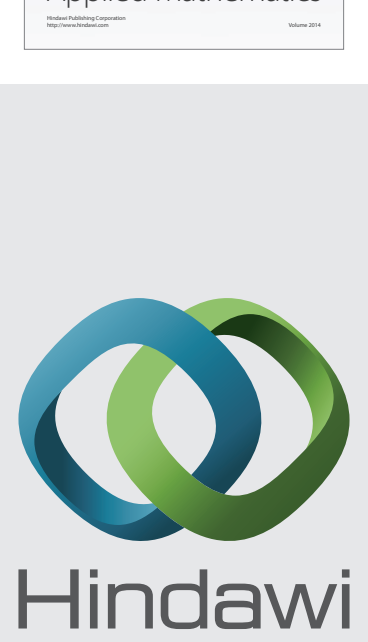

Submit your manuscripts at http://www.hindawi.com
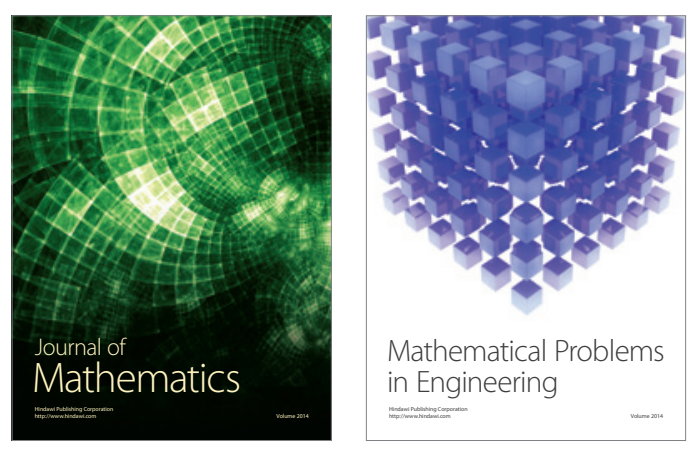

Mathematical Problems in Engineering
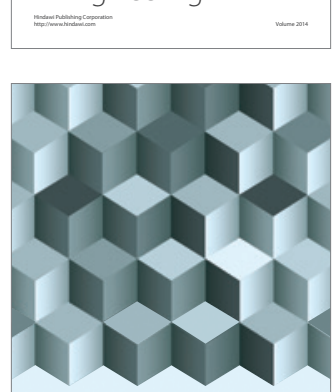

Journal of

Function Spaces
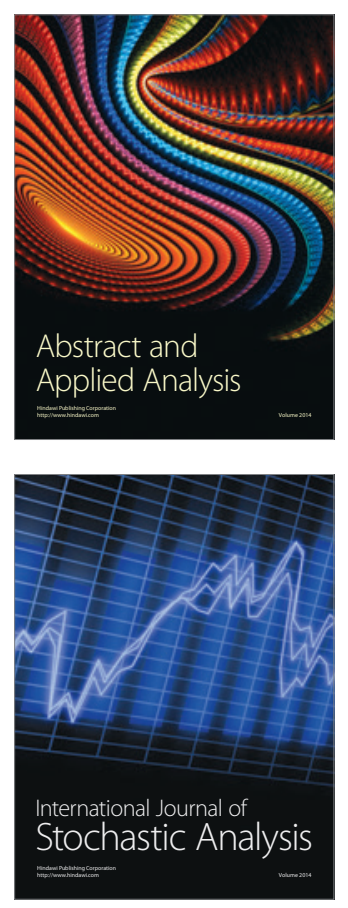

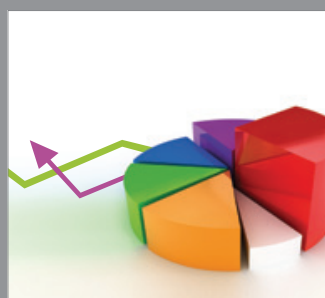

ournal of

Probability and Statistics

Promensencen
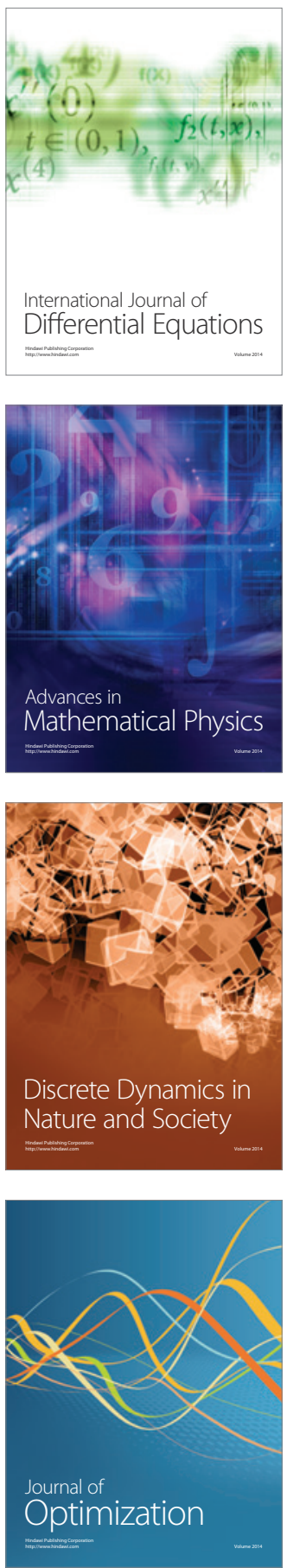